\title{
Milliliter per Hour per Meter Squared
}

National Cancer Institute

\section{Source}

National Cancer Institute. Milliliter per Hour per Meter Squared. NCI Thesaurus. Code C120788.

A unit of concentration equal to milliliter per hour divided by meter squared. 